This document is the Accepted Manuscript version of a Published Work that appeared in final form in British Journal of Midwifery, copyright (C) MA Healthcare, after peer review and technical editing by the publisher. To access the final edited and published work see [DOI]

\title{
Lived experiences of young pregnant women who smoke.
}

\begin{abstract}
Background: Smoking tobacco during pregnancy can lead to adverse pregnancy and child health outcomes.

Aim: The purpose of this research was to gain insight, from young pregnant women's lived experience, of smoking during pregnancy.

Methodology: A descriptive phenomenological approach was used. Semi -structured interviews were conducted with five women between the ages of 18- 20 years who had smoked tobacco during pregnancy.

Results: Themes that emerged from the data were related to: culture and routine, psychological well-being, self-efficacy, public opinion, recognition of harm, who they spoke to about smoking and the approach, what helped behaviour change.

Conclusions: Midwives are seen as the trusted health professional by young women and a non-judgmental approach is valued to promote self efficacy. Smoking cessation services were not recognised as beneficial and cutting down, rather than using Nicotine Replacement Therapy or e-cigarettes was preferred.
\end{abstract}

Fiona Johnson Health Programme Advisor, Public Health Dorset.

Dr Jaqui Hewitt-Taylor Senior Lecturer Bournemouth University,Faculty of Health and Social Sciences, Department of Health Sciences and Public Health.

Dr Elizabeth Norton Senior Lecturer Bournemouth University,Faculty of Health and Social Sciences, Department of Health Sciences and Public Health.

Corresponding author: Fiona Johnson Fiona.johnson@dorsetcouncil.gov.uk 


\section{Keywords}

Young Pregnant women / Smoking tobacco/ Midwives/ Smoking cessation services/ Behaviour change/ Qualitative research

\section{Introduction}

Smoking tobacco during pregnancy contributes to numerous adverse pregnancy outcomes (Salihu and Wilson 2007; Marufu et al. 2015). In addition, early life influences later health outcomes and exposure to problems in foetal growth may lead to higher risks of disease in adult life (Gluckman et al. 2008; Eriksson 2010; Sipola-Leppänen et al. 2015).

Women who smoke during pregnancy tend to be younger and from lower socioeconomic groups (Hiscock et al. 2012; NHS Digital, 2012). Despite a steady year on year decline in the number of pregnant women in England who smoke from $15.8 \%$ in $2006 / 7$ to just under 11\% in 2017/18 (NHS Digital 2018), younger mothers are least likely to stop smoking, with only 38\% reporting any cessation (NHS Digital 2012). Two systematic reviews have found that smoking cessation interventions for pregnant women are effective and significantly reduce smoking in late pregnancy (Cochrane Collaboration 2009; Chamberlain et al. 2013). However it is unclear whether this applies to younger women. Only two studies in the above reviews specifically consider women aged twenty or younger. The first, Albrecht et al (1999), may be less applicable in today's context, although their more recent study (Albrecht et al. 2006) demonstrated that for those aged between 14 and 19 years Cognitive Behaviour Therapy (CBT) combined with peer support from a non-smoking friend had significantly more effect than the usual care provided to the control group.

Flemming et al's (2013) literature review suggests that contextual issues significantly affect smoking in pregnancy. This review included two UK studies of younger women. Firstly, Bryce et al's (2007) mixed method study identified that women aged 25 years and under were nervous of engaging with services, perceiving they would be "told off" for smoking. 
Secondly, Hill et al's(2013) qualitative study found that women aged 16-19 years old preferred to try and give up on their own and distrusted smoking cessation provision.

It is acknowledged that more research is needed concerning the most effective ways of helping pregnant teenagers to stop smoking (NICE 2010, Smoking in Pregnancy Challenge Group 2015). Currently, maternity services provide information and offer smoking cessation support, but this appears not to translate into younger women successfully quitting (Hill et al. 2013). Due to the paucity of evidence and understanding about this age group, gaining an insight into what it is like for a young woman to be pregnant and to smoke tobacco was considered important prior to proposing specific interventions. Consequently this study explored the experiences of young women aged 18-20 years who had smoked tobacco at some point during their pregnancy.

\section{Method}

Creswell (2013) identifies phenomenology as an appropriate methodology for describing individuals' lived experience of a concept or phenomenon. This study used descriptive phenomenology to explore young women's lived experiences of smoking tobacco whilst pregnant.

\section{Ethical approval}

Approval to conduct the study was sought and granted by the relevant University Ethics Committee. The study was conducted in line with the approval granted.

\section{Sample and recruitment}

A purposive sample of young women who had smoked during pregnancy was recruited, as the intention was not to seek representativeness, but, as described by O'Leary (2004) to select a sample for defined purposes. The inclusion criteria for women were:

- $\quad$ aged $18-20$ years 
- $\quad$ pregnant or have given birth within the last year

- $\quad$ smoked tobacco at some point during pregnancy

Creswell (2013) recommends a sample of between 1 and 10 people for phenomenological studies. Five women participated in this study, six had been the desired number but one young woman was unavailable during the interview period.

Recruitment occurred via an organisation that provides a young parent's group to support a healthy pregnancy and beyond. The researcher met personally with a group of young women to explain the research, as it was recognised that this may help to build trust (Robinson 2014). All potential participants received a verbal explanation and a Participation Information sheet clarifying what involvement would entail, and subsequently had time to consider their decision. Those who agreed to participate were asked to complete a Participation Agreement form, and an interview was arranged. Of the five participants three had smoked throughout pregnancy and two had given up when they realised they were pregnant. All participants were white British, engaged with the service via which recruitment took place, and none had planned their pregnancy.

\section{Data collection}

Data were collected using face-to-face semi-structured interviews with open-ended questions. Interviews lasted between 18 - 39 minutes, and, with participants' permission, were audio recorded. One researcher conducted and transcribed verbatim all the interviews, to give continuity, consistency and to allow full engagement in the research topic.

\section{Data analysis}

The data analysis process was based on the Giorgi (2009) approach to descriptive phenomenology. The steps of which are shown in Box 1. 
From the data seven key themes emerged, describing the essence of young women's experiences. The themes and related subthemes are shown in Table 1.

Box 1: Giorgi (2009) approach to descriptive phenomenology.

Step 1: Initial reading of each transcript, noting the overall sense of what was described.

Step 2: Re-reading the transcript, observing and marking each time the dialogue altered in its meaning so as to divide the transcript into a series of meaning units.

Step 3: Examining each meaning unit to discover the important element.

Step 4: Integration of meaning units from across participants and development of key themes.

\section{Findings}

\section{Culture and routine}

Smoking was a key element of participants' social routine and culture: all had smoked for several years, mostly starting at approximately 14 years of age within social gatherings. Smoking was now a part of their daily routine, most of the people they spent time with smoked, they had not previously considered giving up, and some felt that it would be hard to stop smoking after so long:

“I've smoked for so long, that I can't imagine not doing it". P1

\section{Psychological well-being}

The participants who continued to smoke identified it as a stress and anxiety relief mechanism: their "go to" when things were difficult emotionally. They described feeling relaxed or calm when they smoked, and able to cope with difficulties. Some thought that giving up completely would be stressful and harmful for them. 
"It's going to be more stressful for me to try and cut it all out now". P3

\section{Self-efficacy for quitting}

Those who continued to smoke felt that stopping completely would be too difficult to achieve.

“I knew I wasn't going to be a heavy smoker but I knew from day dot that I wasn't going to give up. Cause like otherwise I would have given up already. I knew for a fact that I wasn't going to stop smoking". P3

Women who did not give up smoking did what they considered the next best thing and cut down. They found this hard but spread a low number of cigarettes out until they really needed them.

\section{Public opinion}

All those who smoked throughout pregnancy experienced a strong social pressure not to. Consequently, they did not want to be seen smoking in public, describing an anxious tension from the weight of other people's opinion towards them. Having to deal with this made them want to smoke more:

"If I was out in public I would feel more of the need to smoke but also not want to, cause I wouldn't like people looking at me and thinking oh god she's smoking and she's pregnant like what is she doing, so I kind of wait until we found like a kind of private area or just try and go as long as I could without having one". P2

\section{Recognition of harm}

All the participants recognised that smoking was not good for their babies, and consequently changed their habits in various ways. They recalled receiving information at school, at a first aid course or through TV adverts, which prompted two participants to stop smoking, describing it as a "no, no" from the start. These two young women had strong mental images 
of what was happening to their baby. One describes that she had heard from someone although she did not specify who.

"Smoking while you were pregnant it's like for the baby crushing the umbilical cord for the baby to breath and they said it was something like it can last for 10 minutes." P4

"I just thought like how can I smoke a cigarette knowing that it's the same as crushing it's breathing pipe basically, because when it come out and it was born I wouldn't sit there and put my thumb on its throat for 10 minutes, so why would I smoke a fag cause it's going to do the same thing". P4

The two young women who gave up found it surprisingly easy: something "clicked" and the desire to smoke had gone, before they had contact with any health professionals.

"I think it was quite easy I kind of just had the appeal to stop, the second I found out I was pregnant it didn't appeal to me to have a cigarette, cause I know it is so not healthy for pregnancy and like if I stop it will just be easier, so I stayed away from friends from family from anyone who smoked". P5

Those who continued to smoke described knowing that giving up was in principle a good thing but also feeling that it would cause stress for them and the baby, which was bad. Later on in pregnancy they deemed it better to cut down than give up entirely as this might cause the baby distress.

"It's dangerous to stop smoking completely if you are so far into your pregnancy, sometimes I feel like the baby wants it cause I didn't stop at 5 weeks". P3

Those who cut down felt that smoking one or two cigarettes a day was not harmful.

"I think just one and a half a day; may be one is just alright it's not going to do that much damage, if I'm honest". P3 
All participants described E-cigarettes as harmful in pregnancy, and considered them worse than a cigarette; they had heard this from the vape seller, friends and some health professionals.

"half these products are just replaced by chemicals, to think you are putting into your lungs, to be honest with you l'd rather sit there and smoke a fag, than sit and vape on a cigarette that's got chemicals going into my lungs, I don't like them at all (intake of breath)". P3

"I think they are probably worse than a cigarette cause of what is in them" P5

There was also a mistrust of Nicotine Replacement Therapies (NRT), and no belief that they could work from two of those who smoked.

“It just doesn't work, like all that gum stuff, like you don't chew gum to help you stop smoking, you can feel it in your lungs that you need it, how is chewing a piece of gum going to stop you from smoking?". P3

The two young women who had given up smoking had not used any NRT or approached anyone for help.

\section{Who they spoke to about smoking and the approach.}

The young women were all happy to discuss smoking with their midwives:

"I did speak to them (midwives) a bit about it, like how many I was smoking in a day and they did keep mentioning to me about going to a stop smoking person that comes here I think, not really sure, but I did get the number down and like and then I just never ended up going". P2

All the women received Carbon Monoxide (CO) screening through their midwives and considered this a positive and useful experience. However, the negative side of this was that 
one young woman who had not stopped smoking felt that as her $\mathrm{CO}$ reading had reduced her baby was now adequately protected.

"I'm blowing a 6 and that's classed as a non smoker so it's not like l'm smoking 10 a day and putting my baby at serious harm". P3

\section{What helped behaviour change.}

The scan appointment, when women saw their babies, was a significant time, reassuring them that everything was alright and motivating them to "really cut down" if they were smoking.

"Yes didn't believe I was pregnant till I had my 12 week scan, it made me cut down a lot more than I had already cut down". P1

Three of the participants were offered the opportunity to access a smoking cessation service, whilst two had given up before this was offered. However, only one sought this support, as participants generally wanted to be in control of their situation themselves, and felt that other people could not really help them. Those who gave up smoking achieved this unaided and were proud of it. One woman, who did not give up smoking, explained why she did not want assistance to do so.

“cause it's quite an achievement giving up smoking, so I kind of thought I don't want to do it just cause somebody's telling me to, I want to make that decision and kind of on my own". P2

The only woman who accessed the smoking cessation service found it unhelpful and upsetting:

"It makes you feel more bad than it does help you. It just makes you feel like really horrible, it doesn't help and then you just want a fag, cause you just feel horrible". P1 
Themes and Subthemes

\begin{tabular}{|c|c|c|}
\hline Themes & Sub themes & $\begin{array}{l}\text { Description(descriptions use } \\
\text { the third person in line with } \\
\text { Giorgi (2009). }\end{array}$ \\
\hline \multirow[t]{2}{*}{ 1.Culture and Routine } & $\begin{array}{l}\text { All had smoked for a long } \\
\text { time. }\end{array}$ & $\begin{array}{l}\text { As she had been smoking for so } \\
\text { long she thought it was hard to } \\
\text { stop. }\end{array}$ \\
\hline & $\begin{array}{l}\text { Family smoked and/or } \\
\text { partner }\end{array}$ & Everyone in her family smokes \\
\hline \multirow[t]{2}{*}{ 2. Psychological Well-being } & Linked with stress & $\begin{array}{l}\text { When things are bad she wants } \\
\text { a cigarette and then she feels } \\
\text { calmer which is what she wants. } \\
\text { It would also be very stressful to } \\
\text { try and give up completely now. }\end{array}$ \\
\hline & See cigarettes as needed & $\begin{array}{l}\text { It is what she does that makes } \\
\text { her feel normal; without it she } \\
\text { feels a sense of loss that she } \\
\text { doesn't like. }\end{array}$ \\
\hline \multirow[t]{2}{*}{ 3.Self-efficacy for quitting } & Self efficacy & $\begin{array}{l}\text { She doesn't think she can stop } \\
\text { as it is too hard. She knew she } \\
\text { wasn't going to give up. }\end{array}$ \\
\hline & $\begin{array}{l}\text { Motivation/ desire and } \\
\text { commitment to the decision. }\end{array}$ & $\begin{array}{l}\text { She described giving up as "it } \\
\text { didn't work" and was not seen as } \\
\text { a choice she was personally } \\
\text { making. }\end{array}$ \\
\hline 4.Public opinion & & $\begin{array}{l}\text { Doesn't want to be seen in town } \\
\text { smoking. }\end{array}$ \\
\hline \multirow[t]{3}{*}{ 5.Recognition of harm } & Recognition of harm & $\begin{array}{l}\text { Thinking of the baby and what } \\
\text { he was breathing in made her } \\
\text { stop. }\end{array}$ \\
\hline & Smoke free homes & She never smokes inside \\
\hline & e-cigarettes & $\begin{array}{l}\text { She didn't see the point in e- } \\
\text { cigarettes, worse than cigarettes. }\end{array}$ \\
\hline \multirow[t]{3}{*}{$\begin{array}{l}\text { 6.Who they spoke to about } \\
\text { smoking and the approach. }\end{array}$} & Response to information & $\begin{array}{l}\text { The information given made her } \\
\text { feel horrible at the time and she } \\
\text { didn't like this. Her response was } \\
\text { to look to cigarettes for comfort } \\
\text { from these feelings. }\end{array}$ \\
\hline & $\begin{array}{l}\text { Approach } \\
\text { Including CO screening. }\end{array}$ & $\begin{array}{l}\text { A low CO reading made her feel } \\
\text { better, even though she was } \\
\text { smoking. }\end{array}$ \\
\hline & Valued the Midwife & $\begin{array}{l}\text { She was happy to talk to her } \\
\text { midwife about smoking. }\end{array}$ \\
\hline \multirow{2}{*}{$\begin{array}{l}\text { 7. What helped behaviour } \\
\text { change. }\end{array}$} & Scan appointment & It made her cut down a lot more. \\
\hline & $\begin{array}{l}\text { Feared for the baby, wanted } \\
\text { to protect. }\end{array}$ & $\begin{array}{l}\text { She worried about her having a } \\
\text { still birth or cot death, she } \\
\text { feared the baby not crying when } \\
\text { it was born and something going } \\
\text { wrong and she didn't want to do } \\
\text { anything that might make that be } \\
\text { the case. }\end{array}$ \\
\hline
\end{tabular}




\section{Discussion}

\section{Young women's experiences.}

These young women's experiences indicate that discovering they were pregnant and the 12 week scan appointment were significant times for motivating them to change their smoking behaviour. Previous studies by DiClemente et al (2000) and Orlander et al (2016) have recognised a change of outlook during pregnancy and the opportunity this may offer in relation to smoking cessation. This study additionally suggests that the scan appointment may provide an important specific opportunity to revisit smoking with young women.

Young women in this study valued CO screening, but a perceived low CO reading did not motivate them to stop smoking, instead endorsing an opinion that cutting down was enough. There is a lack of clarity over the CO level commensurate with non-smoking with 3ppm, 4 ppm and 6-10ppm all being cited (NICE 2010, The Smoking in Pregnancy Challenge Group 2016). This suggests that health professionals should follow NICE (2010) guidance and assess smoking through both $\mathrm{CO}$ monitoring and discussion, exploring with women how low CO readings should be interpreted, with reasons for, and implications of, lower readings explained.

\section{Barriers to smoking cessation.}

This study identified psychological well-being as a key factor preventing young women from quitting smoking. Flemming et al's (2015) systematic review also found that women of all ages identify smoking as a stress reliever and a way of coping with anxiety. Whilst young 
pregnant women in this study experienced guilt resulting from perceived social disapproval for smoking, as identified by Flemming et al. (2013); Flemming et al. (2015) and; Bauld et al. (2017), this increased their stress, hindering, rather than enhancing, any attempt to stop smoking. This study also supports Bull et al's (2007) evidence that the stigma of smoking can make women covert about their habits, and harder to reach for the purpose of smoking cessation support.

The women in this study who continued to smoke whilst pregnant described a low selfefficacy in their ability to quit; also identified by Bauld et al (2017) in women of all ages. This suggests that younger women's belief in their own capacity may dictate whether they successfully stop smoking. Understanding and trying to build up young women's self-efficacy could, therefore, be a key element of helping them to stop smoking. Two previous studies suggest that interventions should go beyond behaviour to explore the psychosocial context for the smoker and provide tiered support depending on the need. (Wakschlag et al. 2003; Pickett et al. 2009).

\section{Facilitators of smoking cessation.}

This study, like that of Bauld et al. (2017), indicates that women value and trust their midwifery contact for discussing smoking. As Bauld et al. (2017) identified, understanding the risk of smoking during pregnancy helped some young women in this study to stop smoking. Those who did not were either unaware of the specific risks from continued smoking or diminished them by rationalising their importance with other messages, as has been acknowledged in women of all ages (Flemming et al. 2015). Helping younger women to really understand and perceive the risks of smoking whilst pregnant may, therefore, be helpful. However, for the young women in this study, feeling judged or told what to do was unhelpful and could increase their stress and lessen their likelihood of giving up. The approach taken to discussing smoking cessation with young women is therefore as crucial as the content. 
Despite valuing their midwives' support, the young women in this study did not consider seeking additional smoking cessation support worthwhile, wanting to retain control of their decision making, possibly relating to their self-efficacy and beliefs about stopping smoking. This corroborates Hill et al's (2013) finding that young women did not see smoking cessation services as relevant or beneficial, preferring to quit alone. This suggests, as DiClemente (2016) highlights, that in order to help young women to change what they do, professionals also need to change what they do, and that services need to be proactive, but also nonjudgemental and woman centred.

Nicotine Replacement Therapy (NRT) and E-cigarettes were unacceptable to the young women in this study, with cutting down considered less harmful. These findings reflect those of Bovill et al. (2017) but demonstrate perceptions contrary to current advice that NRT or Ecigarettes have fewer risks for pregnant women than smoking (NHS Choices 2016). Other studies, however, have found that women deem E-cigarettes preferable to smoking during pregnancy (Kahr et al. 2015; England et al. 2016). This inconsistency suggests that further research on the acceptability of E-cigarettes as an alternative to smoking in pregnancy, particularly amongst younger women, is needed. Cutting down is not a recognised harm reduction method in pregnancy and is not mentioned in NHS advice (NHS Choices 2016). Its absence in such guidance may leave women and professionals unclear about the risks associated with smoking small amounts of tobacco, and has implications for helping young pregnant women to make informed decisions.

\section{Strengths}

This study used a recognised phenomenological methodology to give it credibility. It was important to ensure honesty from the participants, to gain an accurate account. The venue was one that provided a private space to talk but was familiar. The researcher made every effort to build a rapport with the participants and used active listening skills. The study has been reported in a clear, systematic and consistent manner in order to provide dependability. 
The confirmability is established as the study provides a data orientated audit trail, showing how the data was gathered and how formulation of the evidence was produced.

\section{Limitations}

It should be recognised that this study is context specific and the intention is that the findings should be applied if the context is comparable. Description of the participants and setting is provided to allow readers to assess its applicability.

In this study a sample of voluntary participants was used and for ethical reasons it was vital that they were willing to contribute. Therefore it is recognised that there will be self selection bias. The researcher was aware that bias could arise in the results from her own assumptions and interpretations. Therefore a reflective journal was used to support this process before and during the study. By using a debrief session between the researcher and supervisors, a level of scrutiny and challenge to bias and preconceptions was provided. Early transcribing of initial interviews was also used as a positive way of helping to assess bias.

\section{Implications for practice}

Midwives play a key role in supporting younger women who smoke during pregnancy, as health professionals who are accepted, trusted and seen regularly (Bauld et al. 2017). Nonjudgmental discussion with young women, exploring self-efficacy, CO monitoring, NRT and e-cigarettes, as well as linking into women-centred smoking cessation services are critical for optimum support.

\section{Conclusion}

Many psychosocial factors hampered young women's ability to stop smoking during pregnancy, with self-efficacy being a key element of successful smoking cessation. Additional smoking cessation services were not valued by young women, and, contrary to current guidelines, cutting down, rather than using NRT or e-cigarettes was considered a 
viable way of minimising harm to the unborn baby. Midwives were seen as the trusted health professional by young women, but a non-judgmental and empowering approach, in which the woman maintains control, needs to be used when addressing smoking. Appreciating the risk for the unborn child helped to facilitate smoking cessation and was the main motivation for those who gave up, with the scan appointment a key time of behaviour change. CO screening was considered helpful although low level results could encourage cutting down instead of stopping smoking.

\section{Key Points}

- Midwives are seen as the trusted health professional by young women and a nonjudgmental approach is valued to promote self efficacy.

- Helping younger women perceive the risks of smoking tobacco whilst pregnant is helpful in supporting a quit attempt but this must be done in a non judgemental empowering manner.

- CO readings need to be discussed carefully so that misleading information is not taken from the screening process.

- Smoking cessation pathways should consider the importance of the 12 week scan appointment for young women and how this can be utilised to support motivation to change smoking behaviour.

- Nicotine Replacement Therapy (NRT) and E-cigarettes were unacceptable to the young women in this study, with cutting down considered less harmful. This demonstrate perceptions contrary to current advice that NRT or E-cigarettes have fewer risks for pregnant women than smoking (NHS Choices 2016). 


\section{CPD reflective questions}

- How would you seek to develop a non-judgmental approach when discussing smoking?

- What do you think could be done to enable young women to gain greater sense of self efficacy?

- How would you explore CO levels with women who had significantly reduced their smoking habit, but had not given up?

\section{List of References}

Albrecht, S. A., Braxter, B., Reynolds, M. D., Stone, C., Cassidy, B. and Cornelius, M. D., 1999. An assessment of nicotine dependence among pregnant adolescents. Journal of Substance Abuse Treatment, 16 (4), 337-344.

Albrecht, S. A., Caruthers, D., Patrick, T., Reynolds, M., Salamie, D., Higgins, L. W., Braxter, B., Kim, Y. and Mlynarchek, S., 2006. A Randomized Controlled Trial of a Smoking Cessation Intervention for Pregnant Adolescents. Nursing Research, 55 (6), 402-410.

Bauld, L., Graham, H., Sinclair, L., Flemming, K., Naughton, F., Ford, A., McKell, J., McCaughan, D., Hopewell, S., Angus, K., Eadie, D. and Tappin, D., 2017. Barriers to and facilitators of smoking cessation in pregnancy and following childbirth: literature review and qualitative study. Health Technology Assessment (Winchester, England), 21 (36), 1-158.

Bovill, M., Gruppetta, M., Cadet-James, Y., Clarke, M., Bonevski, B. and Gould, G. S., 2017. Original Research - Qualitative: Wula (Voices) of Aboriginal women on barriers to accepting smoking cessation support during pregnancy: Findings from a qualitative study. Women and Birth.

Bryce, A., Butler, C., Gnich, W., Sheehy, C. and Tappin, D. M., 2009. CATCH: development of a home-based midwifery intervention to support young pregnant smokers to quit. Midwifery, 25 (5), 473-482.

Bull, L., Burke, R., Walsh, S. and Whitehead, E., 2007. Social attitudes towards smoking in pregnancy in East Surrey: A qualitative study of smokers, former smokers and nonsmokers. Journal of Neonatal Nursing, 13, 100-106.

Chamberlain, C., O'Mara-Eves, A., Oliver, S., Caird, J. R., Perlen, S. M., Eades, S. J. and Thomas, J., 2013. Psychosocial interventions for supporting women to stop smoking in pregnancy [online]. London: Cochrane. Available from: https://www.cochrane.org/CD001055/PREG psychosocial-interventions-supportingwomen-stop-smoking-pregnancy [Accessed 1.4.19] 
Cochrane Collaboration, 2009. Interventions for promoting smoking cessation during pregancy [online]. New Jersey: John Wileyand Sons Ltd. Available from: http://onlinelibrary.wiley.com/doi/10.1002/14651858.CD001055.pub3/epdf [Accessed 20.3.19].

Creswell, J. W., 2013. Qualitative inquiry \& research design : choosing among five approaches [Non-fiction]. Los Angeles, [Calif.] ; London : SAGE, c2013. 3rd ed.

DiClemente, C. C., 2016. Failure to change or failure to sustain: pregnancy smoking and postpartum abstinence. Addiction, 111 (6), 992-993.

DiClemente, C. C., Dolan-Mullen, P. and Windsor, R. A., 2000. The process of pregnancy smoking cessation: implications for interventions. Tobacco Control, 9 Suppl 3, III16III21.

England, L. J., Tong, V. T., Koblitz, A., Kish-Doto, J., Lynch, M. M. and Southwell, B. G., 2016. Perceptions of emerging tobacco products and nicotine replacement therapy among pregnant women and women planning a pregnancy. Preventive medicine reports, Vol 4, Iss C, Pp 481-485 (2016), (C), 481.

Eriksson, J. G., 2010. Early programming of later health and disease: factors acting during prenatal life might have lifelong consequences. Diabetes, 59 (10), 2349-2350.

Flemming, K., Graham, H., Heirs, M., Fox, D. and Sowden, A., 2013. Smoking in pregnancy: a systematic review of qualitative research of women who commence pregnancy as smokers. Journal of Advanced Nursing, 69 (5), 1023-1036 1014p.

Flemming, K., McCaughan, D., Angus, K. and Graham, H., 2015. Qualitative systematic review: Barriers and facilitators to smoking cessation experienced by women in pregnancy and following childbirth. Journal of Advanced Nursing, 71 (6), 1210-1226.

Giorgi, A., 2009. The descriptive phenomenological method in psychology : a modified Husserlian approach. Pittsburgh, Pa.: Duquesne University Press.

Gluckman, P. D., Hanson, M. A., Cooper, C. and Thornburg, K. L., 2008. Effect of in utero and early-life conditions on adult health and disease. New England Journal of Medicine, 359 (1), 61-73 13p.

Hill, S., Young, D., Briley, A., Carter, J. and Lang, R., 2013. Baby Be Smoke Free: Teenage smoking cessation pilot. British Journal of Midwifery, 21 (7), 485.

Hiscock, R., Bauld, L., Amos, A. and Platt, S., 2012. Smoking and socioeconomic status in England: the rise of the never smoker and the disadvantaged smoker. Journal of Public Health, 34 (3), 390-396 397p.

Kahr, M. K., Padgett, S., Shope, C. D., Griffin, E. N., Xie, S. S., Gonzalez, P. J., Levison, J., Mastrobattista, J., Abramovici, A. R., Northrup, T. F., Stotts, A. L., Aagaard, K. M. and Suter, M. A., 2015. A qualitative assessment of the perceived risks of electronic cigarette and hookah use in pregnancy. BMC Public Health, 15 (1), 1. 
Marufu, T. C., Anand, A., Coleman, T. and Lewis, S., 2015. Maternal smoking and the risk of still birth: systematic review and meta-analysis. BMC Public Health, 15 (239)..

NHS Choices, 2016. Stop smoking in pregnancy [online]. London: Available from: http://www.nhs.uk/conditions/pregnancy-and-baby/pages/smoking-pregnant.aspx [Accessed 1.4.19].

NHS Digital, 2012. Infant Feeding Survey 2010, [online]. Leeds,: NHS Digital. Available from: https://files.digital.nhs.uk/publicationimport/pub08xxx/pub08694/ifs-uk-2010-chap11diet-supp-smok-drink.pdf [Accessed 1.4.19]

NHS Digital, 2018. Statistics on women's smoking status at time of delivery, England-quarter 4, 2017-18,[online].Leeds: NHS Digital. Available from: https://digital.nhs.uk/dataand-information/publications/statistical/statistics-on-women-s-smoking-status-at-timeof-delivery-england/statistics-on-womens-smoking-status-at-time-of-delivery-england--quarter-4-october-2017-to-december-2017/part-2 [Accessed 1.4.19].

NICE, 2010. Smoking:stopping in pregnancy and after childbirth. PH26 [online].London NICE Guidance. Available from: https://www.nice.org.uk/guidance/ph26 [Accessed 1.4.19]. O'Leary, Z., 2004. The Essential Guide To Doing Research. London: Sage.

Olander, E. K., Darwin, Z. J., Atkinson, L., Smith, D. M. and Gardner, B., 2016. Beyond the 'teachable moment' - A conceptual analysis of women's perinatal behaviour change. Women \& Birth, 29 (3), e67.

Pickett, K. E., Wilkinson, R. G. and Wakschlag, L. S., 2009. The psychosocial context of pregnancy smoking and quitting in the Millennium Cohort Study. Journal of Epidemiology and Community Health (1979-), (6), 474.

Robinson, O. C., 2014. Sampling in interview-based qualitative research: A theoretical and practical guide. Qualitative Research in Psychology, 11 (1), 25-41.

Salihu, H. M. and Wilson, R. E., 2007. Epidemiology of prenatal smoking and perinatal outcomes. Early Human Development, 83 (11), 713-720.

Sipola-Leppänen, M., Vääräsmäki, M., Tikanmäki, M., Matinolli, H. M., Miettola, S., Hovi, P., Wehkalampi, K., Ruokonen, A., Sundvall, J., Pouta, A., Eriksson, J. G., Järvelin, M. R. and Kajantie, E., 2015. Cardiometabolic risk factors in young adults who were born preterm. American Journal of Epidemiology, 181 (11), 861-873.

Smoking in Pregnancy Challenge Group, 2015. Smoking Cessation in Pregnancy: A call to action [online]. London: ASH. Available from: http://ash.org.uk/information-andresources/smoking-cessation-treatment/smoking-cessation/smoking-cessation-inpregnancy-a-review-of-the-challenge/ [Accessed 20.3.19].

Smoking in Pregnancy Challenge Group.2016 Carbon Monoxide screening. [online] 
London:Smokefree Action Coalition. Available from

http://smokefreeaction.org.uk/smokefree-nhs/smoking-in-pregnancy-challenge-

group/smoking-in-pregnancy-challenge-group-resources/carbon-monoxide-

screening/ [Accessed 18 June 2019]

Wakschlag, L. S., Pickett, K. E., Middlecamp, M. K., Walton, L. L., Tenzer, P. and Leventhal,

B. L., 2003. Pregnant smokers who quit, pregnant smokers who don't: does history of problem behavior make a difference? Social Science \& Medicine (1982), 56 (12),

$2449-2460$ 